\title{
Renovación de la acreditación de alta calidad para el programa de mercadeo y publicidad
}

\section{Un logro producto de la madurez que da la experiencia de lo que ha sido bien construido}

Como Decano de la Facultad de Mercadeo, Comunicación y Artes me llena de inmensa alegría comunicarle a toda la comunidad académica la obtención de la renovación de la acreditación de alta calidad que cavamos de lograr para el programa profesional en Mercadeo y Publicidad expedida por el Ministerio de Educación Nacional en Diciembre de 2012.

Indudablemente, este logro hace parte de todo el equipo académico que actualmente me acompaña en la Facultad pero el mismo sólo puede ser fruto de buenas siembras y buenas cosechas en las que han intervenido nuestros antecesores.

Por eso debo empezar por hacer un importante reconocimiento a quienes me antecedieron en este cargo y estuvieron al frente del programa de Mercadeo y Publicidad entre 1986 y 2010: a Jurgen Chiari Escovar porque le dio toda la estructura académica de base, porque integró el Mercadeo y la Publicidad en un sólo programa, porque creó el ciclo profesional como segundo ciclo del programa de Tecnología en Mercadeo y Publicidad.

A Juanita Michelsen Niño porque lo acreditó internacionalmente ante la International Advertising Association IAA, porque modernizó la visión del Mercadeo como generación de valor en la cadena de producción/consumo, porque lo internacionalizó con la realización del primer Encuentro de Facultades de Mercadeo y Publicidad de América Latina, porque lo volvió más integral con sus proyectos del Centro de Gestión de la Información dándole valor investigativo y de proyección social, porque lo acredito como un programa de alta calidad en el ámbito nacional y porque fue una de las importantes artífices de la renovación que hoy celebramos. 
A Carlos Augusto García López porque lo integró a la nueva Facultad de Mercadeo, Comunicación y Artes en conjunción con los programas de Comunicación Social Periodismo y de Medios Audiovisuales, y porque lideró la reforma curricular del año 2008, que fue la base para obtener este segundo reconocimiento por parte del Consejo Nacional de Acreditación.

Ahora bien, estamos hablando de la organización, la administración y la gestión de un programa que está integrado por los estudiantes, los docentes, los graduados y los colaboradores de la Institución, que también son responsables de los éxitos alcanzados, los cuales fueron posibles gracias a su participación, colaboración, responsabilidad e interés.

Se hace necesario hablar de los apoyos incondicionales de los Rectores de la Institución en las diferentes etapas del programa, doctores César Tulio Delgado Hurtado, Pablo Michelsen Niño y Fernando Dávila Ladrón de Guevara; hay que reconocer nombres que contribuyeron curricular y didácticamente con la acreditación alcanzada, disculpándome por los seguros olvidos, recuerdo hoy a Jorge Molina
Villegas, Daniel Ronderos, Socorro Jaramillo, Andrés Villegas, Samir Kihuan, Ricardo Martínez, Juan Pablo Rocha, Rubén Darío Gómez, Catalina Mesa, Dora Elvira Acero, Martha Restrepo, Daniel Montes, Lucía Cadena, José Ricardo Franco, Jorge Garcés, Mónica Baquero, Diana Forero, Jose Tiusabá, Edilma Pineda y Mónica Vargas, en las diferentes épocas.

Hay que hablar de otros apoyos esenciales como los que académica, logística u operativamente nos han prestado Rita Robert (q.e.p.d), Angel Beccassino, Edgar Samudio, Martha Eugenia Ruíz, Hugo Colmenares, María Inés Delgado, Camilo García, Fernando Vélez, Carlos Serna, Luis Guillermo Castellanos, Edgar Gualteros, Rodrigo Ferro, Hugo Mastrodoménico, John Jaime Marín, Enrique Coral, Juan Carlos Rivera y Carmen Orozco, entre otros.

Muchas veces, como se dice coloquialmente, hemos sacado pecho, tomando como referentes de nuestros graduados a Miguel de Narváez, Presidente de Sonido comercial, Álvaro José Fuentes, Presidente de McCann Erickson Corporation, Enrique Muñoz, Director del Programa de Ingeniería de Mercados de la Universidad Piloto, de Hugo 
Corredor, Vicepresidente Creativo de Sancho BBDO, Rodrigo Torres, Vicepresidente Creativo de JWT, Juan Martín Borrero, Director de Mercadeo de HP para América Latina, John Conta, Director Comercial de Fruco; Neil Rodrigo Jiménez Cifuentes, Director de Posgrados de la UAO, Francisco Soto, Director CRM Leo Burnett, Luis Miguel Prieto, Gerente General Leo Burnett Guatemala, Álvaro De Luna, Gerente de marca de Miller Bavaria, Faryd Castillo Santiago, Gerente de Marketing Bagó, Salud, Alberto Osorio reyes, Gerente General de Óptima TM, Jaime Vásquez, Gerente Genoma Lab, Federico Estrada Fernández, Gerente $e$-commerce Carrefour, Juan Felipe Velandia, Director General Treetop Rocket S.A., Juan Pablo García, Creativo Lowe y ganador de Premio Cannes 2011, María Constanza Santacruz, Premio globo 2012, Álvaro Potes, Director General On Brand Experiencie, Camilo Plazas, Director Genetral Why not Challenge, Madrid, España, María Isabel linero, Directora Mercadeo Social de RCN Y Juanita Del Corral, Gerente General de Ogilvy and Mather S.A., entre otros, a quienes no podíamos dejar por fuera de estas breves notas y expresarles todo nuestro agradecimiento (datos a noviembre 2012).

Lógicamente, no podía dejar por fuera de este reconocimiento al equipo del programa que actualmente acompaña mi gestión, principalmente a Edwin Leonardo Ortegón Cortazar, docente del Departamento de Mercadeo, que coordinó todo el proceso, elaboró los informes finales y respondió por los resultados ante la Institución y ante los pares académicos del CNA; a Javier Darío Jiménez Herrera Director del Departamento, César Atehortua, coordinador del área de Publicidad, Ricardo Vega, coordinador de Investigación y Posgrado, Rodolfo Vargas Vallecilla, coordinador de Educación Virtual, Sandra González Coordinadora de Prácticas y los docentes de planta Aida Ximena León, César Erazo, Julián Arias y Anie Rodríguez.

A los directores de los Departamentos de Comunicación, Nancy Ballestas, de Diseño y Arte, Leonardo Paez, de Medios Audiovisuales, Harvey Murcia, de Ciencias Básicas, Hugo Zamora, de Humanidades, Santiago Castro, de Derecho y Ciencia Política, Ángela Gómez, de Economía y Finanzas, Deisy De la Rosa, de Administración, Consuelo Torres y de Idiomas, Raquel Bretón. 
Valgan pues estas líneas para de quienes hemos aprendido no celebrar al tiempo que agradecer sólo la metodología para los procea todos aquellos que de una u otra sos de autoevaluación, sino el valor manera han contribuido a cons- de la misma en esa otra parte que truir esta realidad de la renovación tiene la Academia y que va más allá de la acreditación de alta calidad de la relación estudiante - docente para el programa de Mercadeo y en el aula de clase.

Publicidad.

Y dejo estas últimas líneas para Sergio Hernández MuÑoz, hacer un reconocimiento especial a Mayo 2013 María Dolores Pérez y Ana Cristina Miranda, asesoras de la Institución, 